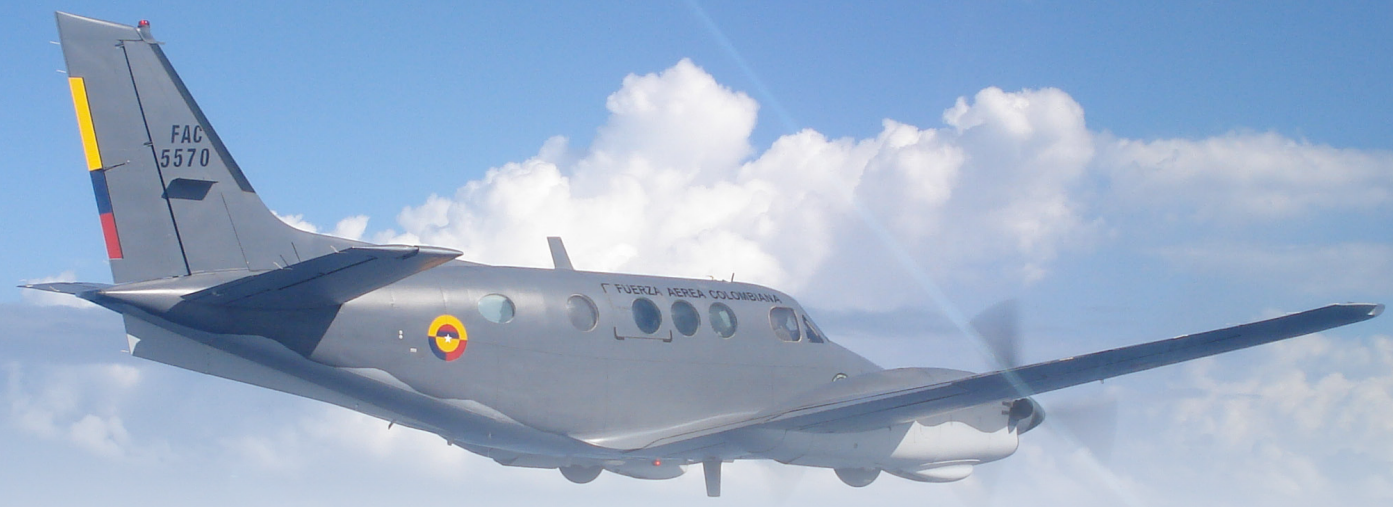

\title{
Prototipo de electrocardiógrafo bipolar para uso académico
}

\author{
Prototype of Bipolar Electrocardiograph for Academic Use ${ }^{2}$ \\ Leonardo Juan Ramírez López ${ }^{3}$ | Yohanna Patricia Rodríguez Ortega ${ }^{4}$ \\ | Yuli Paola Cifuentes Sanabria ${ }^{5}$
}

\begin{abstract}
CIENCIA Y PODER AÉREO
ISSN 1909-7050 | E-ISSN 2389-2468 |Volumen 9 | Enero-Diciembre de 2014 | Colombia | Pp. 115-123

Recibido: 24/11/2014

Aprobado evaluador interno: 27/11/2014

Aprobado evaluador externo: 10/12/2014
\end{abstract}

Artículo científico original, que recoge los resultados de investigación sobre el diseño de electrocardiógrafos de bajo costo para usos académicos, correspondiente a de la línea de investigación de Telemetría del grupo de Investigación en Telemedicina de la Universidad Militar Nueva Granada TIGUM-, financiado por la Universidad Militar Nueva Granada PIC-ING-1668

2 Original scientific article, gathering research results on the design of low cost electrocardiograph for academic uses, from the research area on Telemetry of the research group in Telemedicine of the Nueva Granada Military University-TIGUM, funded by Nueva Granada Military University PIC-ING-1668.

${ }^{3}$ Ingeniero Electrónico, Magíster en Ing. Sistemas y Doctor en Ing. Biomédica. Líder del Grupo de Investigación en Telemedicina de la UMNG - TIGUM, Bogotá, Colombia. Correo electrónico: tigum@unimilitar.edu.co

Electronic Engineer, Master in Systems Engineering and PhD in Biomedicine. Lead of the Research Group in Telemedicine of the UMNG-TIGUM, Bogota, Colombia. Email: tigum@unimilitar.edu.co

${ }^{4}$ Estudiante de Ingeniería en Telecomunicaciones de la UMNG. Semillero Ginko del Grupo de Investigación en Telemedicina de la UMNG - TIGUM, Bogotá, Colombia. Correo electrónico: tigum@unimilitar.edu.co

Telecommunications Engineering Student of the UMNG. Ginko Hotbed of research of the Research Group in Telemedicine of the UMNG-TIGUM, Bogota, Colombia.E-mail:tigum@unimilitar.edu.co

${ }^{5}$ Ingeniera Industrial, Joven investigadora del Grupo de Investigación GISSIC de la UMNG, Bogotá, Colombia. Correo electrónico: cifuentes.yuli@gmail.com

Industrial Engineer, Young Researcher at GISSIC research group of the UMNG, Bogota, Colombia, E-mail:cifuentes.yuli@gmail.com
Resumen: Para la preparación del personal médico en la adquisición, procesamiento, visualización y almacenamiento de señales cardíacas, la tecnología ofrece una serie de equipos y dispositivos de alta calidad y alto costo, además de ser ensamblados de forma compacta que impide entender el procesamiento de la señal a nivel interno. Es por esto, que el Grupo de Investigación en Telemedicina de la Universidad Militar Nueva Granada consiente de la importancia de brindar herramientas de aprendizaje de equipos médicos ha diseñado un electrocardiógrafo para uso académico con tecnología nodular de bajo costo. Los resultados alcanzados con estudiantes de medicina, de electrónica, de biomédica y de telecomunicaciones son altamente satisfactorios y les ha permitido ensamblar y desensamblar el prototipo de electrocardiógrafo diseñado, hacer prácticas de caza-fallas y cumplir con el objetivo de la metodología aprender-haciendo la adquisición, procesamiento, visualización y almacenamiento de señales cardiacas.

Palabras clave: Académico, bipolar, digital, electrocardiógrafo.

Abstract: For preparation of medical and biomedical personnel in the acquisition, processing, display and storage of cardiac signals, the technology offers a number of computers and devices of high quality and high cost, besides being assembled compactly which prevents from understanding the internal signal processing. It is for this reason that the Telemedicine Research Group of Nueva Granada Military University, aware of the importance of providing learning tools for medical equipment has designed an electrocardiograph for academic use with low-cost nodular technology. The results achieved with medical, electronics, biomedical and telecommunications students are highly satisfactory and have allowed them to assemble and disassemble the electrocardiograph, by troubleshooting practices and fulfilling the objective of the methodology of learning by doing, to master the acquisition processing, display and storage of cardiac signals.

Key Words: Academic, Bipolar, Digital, Electrocardiograph. 


\section{Introducción}

La preocupación del ser humano por tener una mejor calidad de vida, está principalmente ligada al estado de su salud, para la emisión de un diagnóstico es indispensable tener un conocimiento certero del funcionamiento del organismo. Actualmente, el personal médico ha encontrado que un diagnóstico a tiempo ayuda a prevenir y tratar enfermedades satisfactoriamente. Haciendo necesario el uso de sistemas electrónicos para la adquisición, procesamiento, transmisión, recepción y visualización de bioseñales que posteriormente son analizadas adecuadamente por el personal idóneo en el campo de la medicina. El constante desarrollo tecnológico que presenta nuestro país ha contribuido a mejorar los equipos utilizados para adquisición de señales y a facilitar su manejo.

En el ámbito investigativo y académico el estudio del funcionamiento del corazón es de gran importancia, debido a que este órgano muscular, hueco en su interior y aproximadamente del tamaño de un puño, es la bomba muscular reforzada más potente que promueve él envió de la sangre por todo el organismo. La mitad derecha de este órgano recibe sangre desoxigenada y la impulsa hacia los pulmones, el lado izquierdo recibe sangre oxigenada de los pulmones, para impulsarla por la aorta y por otras cavidades de comunicación, para posteriormente la trasmitirla por todo el cuerpo ( $\mathrm{L}$ Finance, 1975). El corazón se contrae unas setenta veces por minuto, late más de cien mil veces al día, durante todos los días de una vida, motivos que han incentivado a la ciencia y la tecnología la creación de dispositivos que permitan conocer a profundidad este órgano.

La investigación electrocardiográfica se inició en el mundo hacia el siglo XIX, con la aparición del electrómetro capilar, después, Willem Einthoven (1905) desarrolló el galvanómetro de cuerda (Acierno, 1994) proyecto que dio pie al inicio electrocardiográfico en el mundo, y en la actualidad representa una de las principales herramientas de la medicina moderna.

El electrocardiógrafo es un dispositivo electrónico que permite realizar un electrocardiograma (ECG), examen que registra la actividad eléctrica del corazón durante un latido mostrando la magnitud y dirección de las señales eléctricas producidas por el corazón, además evalúa el ritmo y la regularidad de los latidos. El corazón emite una señal eléctrica que tiene una amplitud que está dada en milivoltios (mV), en un intervalo de entre $0.5 \mathrm{mV}$ a $2 \mathrm{mV}$; además posee ondas características que se producen a partir de las variaciones de potencial eléctrico durante el ciclo cardiaco (Arango, Vélez, Rojas, Borrero, y Restrepo, 2003)

Las ondas características del ECG son: la "onda P", la cual indica que las aurículas son estimuladas de forma eléctrica para enviar la sangre hacia los ventrículos. Posteriormente se realiza una elevación mayor unida a dos caídas que en conjunto que se conoce como el "complejo QRS"; indicando que los ventrículos son estimulados eléctricamente para bombear la sangre hacia fuera (Correa, Bolaños, y Escobar, 2007). El próximo segmento corto, prácticamente plano ascendente se llama "segmento ST". Este indica la cantidad de tiempo que ocurre desde el final de una contracción de los ventrículos hasta el comienzo del periodo de reposo. La onda siguiente al "segmento ST" es ascendente la cual se denominó "onda T"; esta muestra el periodo de recuperación de los ventrículos. Las duraciones normales de las ondas P, el complejo QRS y T son: 0.11 segundos, 0.09 segundos, 0.15 segundos respectivamente; lo que origina anchos de banda por segmentos de $9 \mathrm{~Hz}, 11 \mathrm{~Hz}$ y 6.6 Hz (Arango, Vélez, Rojas, Borrero, y Restrepo, 2003).

En el mundo existen varios desarrollos tecnológicos enfocados en la adquisición de la señal electrocardiográfica, dentro de estos se encuentra el diseño del señor Claudius Moor ${ }^{6}$, quien en el año 2004 desarrollo un módulo denominado OEM (tablero de circuito impreso de ECG), el cual permite visualizar el electrocardiograma desde diferentes tipos de conexiones o con puerto USB. En ese mismo año el Ing. José Manuel Rodríguez utilizó la tecnología Bluetooth y GSM (Global System for Mobile Communications) como una solución integrada para enviar el electrocardiograma y otros registros acerca del estado actual del paciente.

Actualmente, lo currículos de las áreas de ingeniería electrónica, eléctrica, biomédica, telecomunicaciones, sistemas, entre otras, y las áreas de las ciencias de la salud tienen asignaturas de instrumentación biomédica en la cual los estudiantes aprenden el uso de equipos tales como electrocardiogramas, electroencefalogramas, monitores, medidores de parámetros fisiológicos

${ }^{6}$ Claudios Moor: Ingeniero alemán diseñador del módulo OEM (tablero de circuito impreso de ECG) quien de manera eficiente implemento en su país un módulo de comunicaciones inalámbrico con tecnología Bluetooth para la comunicación de ECG.

7 José Manuel Rodríguez Ascariz: Ingeniero en Telecomunicaciones y PhD de la Universidad Politécnica de Madrid, España, en 1998 al 2002. Él es actualmente profesor auxiliar en la Universidad de Alcalá, España. 
entre otros. El aprendizaje es netamente teórico y en muy pocos casos cuentan con estos equipos para hacer pruebas de funcionamiento y conectividad, esto debido principalmente al costo elevado. Es necesario que las instituciones de educación técnica, tecnológica y universitaria cuenten con diseños de prototipos funcionales que logren suplir la necesidad del estudiante de no solo manipular el equipo sino comprender su operatividad interna, modos de procesamiento y tratamiento de las señales, calibración y ajuste.

Actualmente, en Colombia el nivel de estudios electrocardiográficos se ha incrementado en la última década, especialmente en ciudades alejadas, las instituciones de educación e instituciones médicas cuentan con equipos altamente costosos para las prácticas relacionadas a la toma y registros de señales e imágenes médicas, esta necesidad de realizar equipos médicos de bajo costo, modulares y de fácil ensamblaje ha motivado al grupo de investigación en telemedicina -TIGUM a diseñar e implementar un prototipo de ECG que utilice diferentes tecnologías comerciales y garantice la facilidad de ensamble, comprensión de los puntos y señales de prueba y además sea herramienta académica de profesores que de forma didáctica puedan mostrar y explicar la adquisición, pre-procesamiento, procesamiento y visualización de las señales electrocardiográficas.

\section{Materiales y método}

Basados en el desarrollo actual de los dispositivos electrónicos para la adquisición de la señal electrocardiográfica se definió los siguientes materiales y el pro- cedimiento a desarrollar con el objetivo de realizar el dispositivo funcional.

\section{Materiales}

Sistema de adquisición

Para la elaboración del sistema de adquisición académico se utilizó la plataforma libre Arduino ${ }^{\circledR}$, basada en un microcontrolador que facilita el desarrollo de diferentes aplicaciones. Arduino ${ }^{\circledR}$ también desarrolla objetos interactivos, debido a que posee sensores, entradas y salidas tanto analógicas como digitales, entre otros. En la Figura 1 se muestra la tarjeta Arduino ${ }^{\circledR}$ Mega ADK, la cual permite accesar mediante 16 entradas análogas $A_{0}$ a $A_{15}$, salidas digitales, puertos de comunicación para programación en C++ o Java. La selección esta tarjeta se da por las siguientes características.

Características Arduino ${ }^{\circledast}$ Mega ADK:

- Está basada en el microcontrolador ATmega2560.

- Interfaz USB para comunicación con teléfonos Android.

- 54 entradas y salidas digitales

- 16 entradas análogas

- 4 UARTS

- Oscilador de $16 \mathrm{MHz}$

- Conexión USB para comunicación con computador y programación

- Entrada de alimentación

- Botón de reset.

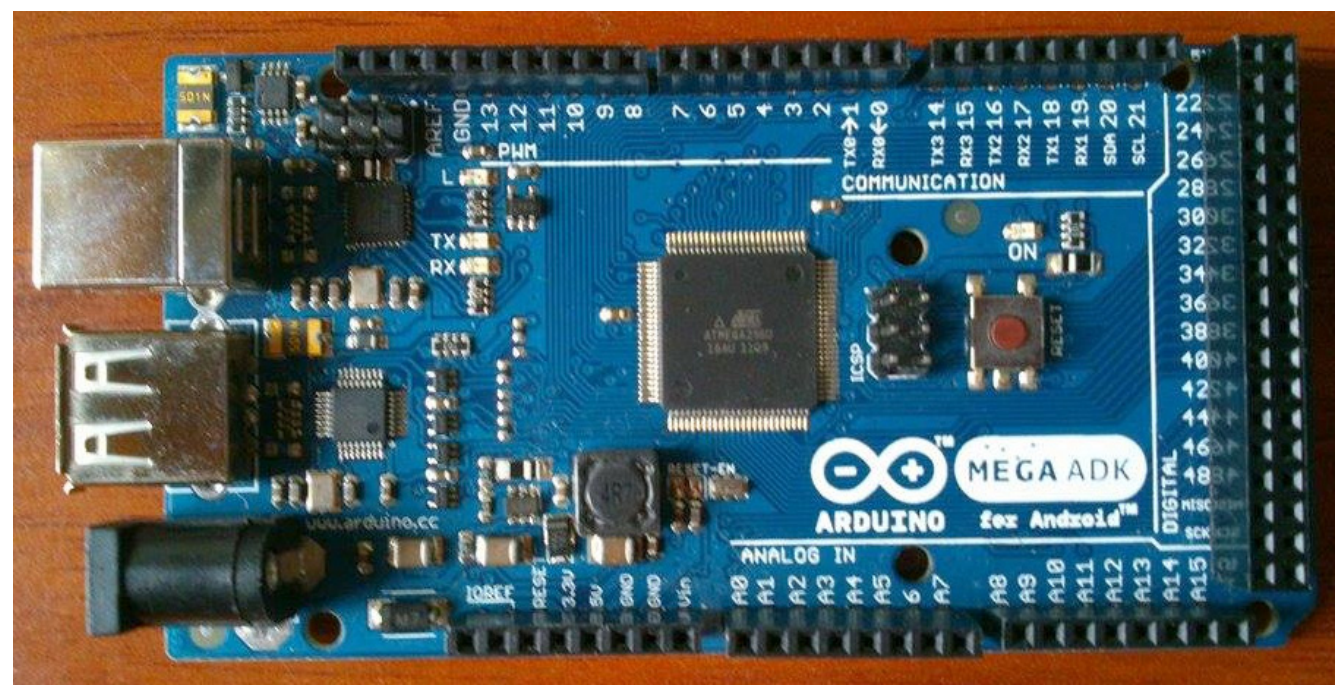

Figura 1. Procesador Arduino ${ }^{\circledR}$ Mega ADK.

Fuente: los autores. 
CIENCIA Y PODER AÉREO | Revista Científica de la Escuela de Postgrados de la Fuerza Aérea Colombiana | Vol. 9 | Enero - Diciembre de 2014

Simulador de paciente FLUKE Biomedical PS420

Este dispositivo utilizado permite simular señales ECG, respiración, presión sanguínea, temperatura y gasto cardíaco de una persona." "El PS420 funciona con baterías y ofrece salida de 12 conductores ECG. La simulación se realiza a través de dos canales de presión sanguínea, con la opción de 35 selecciones de arritmia, simulación de marcapasos y ritmos sinusales normales tanto adultos como pediátricos" (Fluke Biomedical PS420, 2014). La Figura 2 muestra el simulador Fluke PS420 que es usado para simular digitalmente señales de 12 derivaciones cardiacas, respiración y algunas anomalías rítmicas del corazón. Este equipo es de vital importancia al momento de experimentar con prototipos biomédicos para tener señales de referencia, protección a paciente real y hacer variaciones de prueba que permitan hacer rediseños al prototipo propuesto.

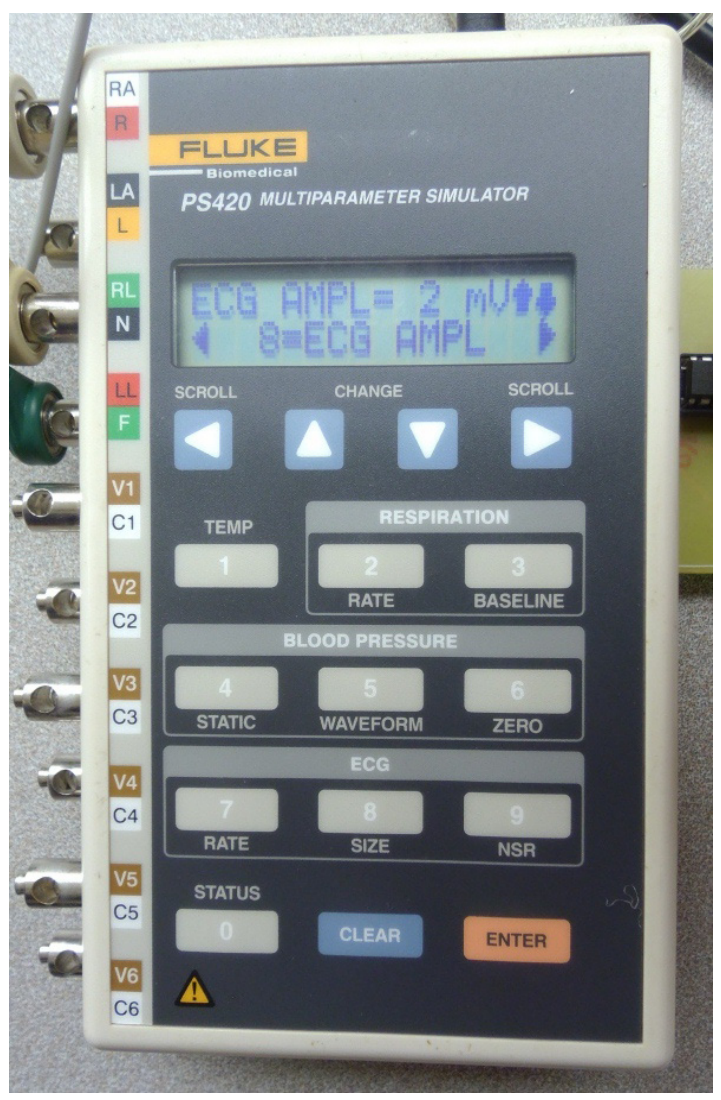

Figura 2. Simulador de paciente FLUKE Biomedical PS420.

Fuente: Los autores.

Características del simulador:

- Electrocardiograma de 12 derivaciones

- Respiración

- Temperatura

- Ritmos sinusales de adultos y pediátricos
- Puerto RS-232 para comunicación serial con computador.

- Utiliza baterias y también alimentación con cable

En la Tabla 1 se presentan algunas especificaciones técnicas del simulador de paciente Fluke PS420:

Tabla 1. Especificaciones técnicas Simulador de paciente FLUKE Biomedical PS420

\begin{tabular}{|c|c|}
\hline \multirow{4}{*}{ 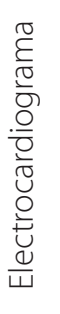 } & Registro normal: 80 PPM \\
\hline & $\begin{array}{l}\text { Registros seleccionables: 30, 40, 60, 80, 100, } \\
\text { 120, 140, 160, 180, 200, 220, 240, 260, } 280 \text { y } \\
300 \mathrm{BPM}\end{array}$ \\
\hline & Precisión: 1\% \\
\hline & Amplitudes ECG: $0.5 \mathrm{mV}, 1 \mathrm{mV}, 1.5 \mathrm{mV}$ y $2 \mathrm{mV}$ \\
\hline \multirow{7}{*}{ 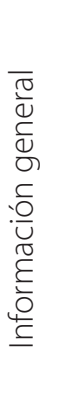 } & Alimentación: 9V \\
\hline & Caja de plástico ABS \\
\hline & Pantalla LCD 2x16 y teclado \\
\hline & RS-232: Interfaz bidireccional de 9600 bauds \\
\hline & Requisitos de temperatura: \\
\hline & Funcionamiento: $15-35^{\circ} \mathrm{C}$ \\
\hline & Almacenamiento: $0-50^{\circ} \mathrm{C}$ \\
\hline
\end{tabular}

Fuente: PS420 Simulador de Paciente. Recuperado el 25 de noviembre de 2014. [En línea] Disponible en http://es.flukebiomedical.com/biomedical/ usen/Biomedical/FB-SIMS/PS420.htm?PID=56628.

\section{Procesamiento}

El montaje y la simulación de los circuitos utilizados en este proyecto se realizaron previamente en el simulador de circuitos (PROTEUS ISIS52, 2008), Software de diseño electrónico desarrollado por Labcenter Electronics que consta de dos módulos: ISIS Y ARES.

ISIS: es un módulo para el diseño de circuitos, que cuenta con una lista muy completa de componentes eléctricos y electrónicos, activos y pasivos, microprocesadores y microcontroladores, estos pueden ser simulados en tiempo real con un módulo VSM, permitiendo simular circuitos con microcontroladores conectados a distintos dispositivos. Además, el modulo ISIS cuenta con herramientas para la realización de esquemas electrónicos (Edminister, Nahvi, Navarro, Sánchez, y de Miguel Rodríguez, 1997).

ARES: es la herramienta de ruteado de PROTEUS, se utiliza para la fabricación de placas de circuito impreso. 
En la Figura 3 se muestra un Print-Script del circuito en PROTEUS ISIS (Ediminister, Nahvi, Navarro, Sánchez, y de Miguel Rodríguez, 1997), herramienta que permite desarrollar diseños electrónicos diversos, probarlos y obtener salidas muy aproximadas a los montajes reales. Se muestra el diseño del sisterma de amplificación de la señal cardiaca de 1:5000 que permita aplicar los filtros digitales o analogos según sea necesario.

La herramienta permite adicionar dispositivos electronicos, procesadores, alimentación y circuitos integrados comerciales para simulación su operación y así determinar su comportamiento al momneto de ensamblar el prototipo de electrocardiografo bipolar.

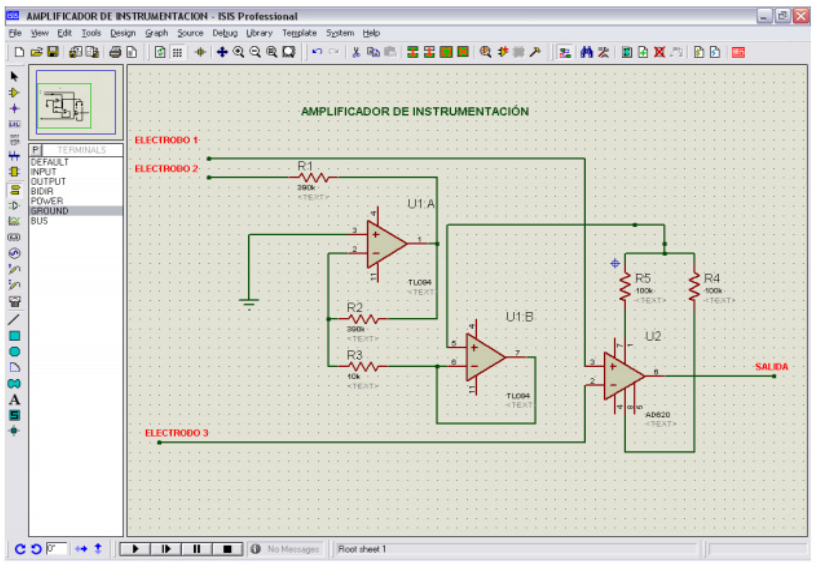

Figura 3. Script PROTEUS ISIS52.

Fuente: elaboración de los autores.

Después de realizar la simulación en PROTEUS, se procede a desarrollar el montaje, conectando el simulador de paciente a la entrada amplificadora de los niveles de voltaje provenientes de la señal cardiaca, debido a que esta señal posee valores bajos de voltaje que son difícilmente procesables por la tarjeta Arduino ${ }^{\oplus}$, además existen voltajes negativos que necesitan ser ajustados en un circuito nivelador DC. Finalmente la señal que se transmite al Arduino ${ }^{\circledR}$ está amplificada, filtrada y es positiva en su rango.

\section{Método}

En la Figura 4 se muestran las etapas de la metodología utilizada en el proyecto que incluye tres etapas: la primera comprende un estudio y tratamiento de la señal de electrocardiografía en donde se define la forma de adquisición, valores de voltaje y resistencia; la segunda atapa de pre-procesamiento que determina la forma de amplificar la señal, filtrarla y entregarla al procesador; en la etapa tres se procesa digitalmente, se almacena y a través de los diferentes puertos de comunicación enviarla a donde el diseñador estime.

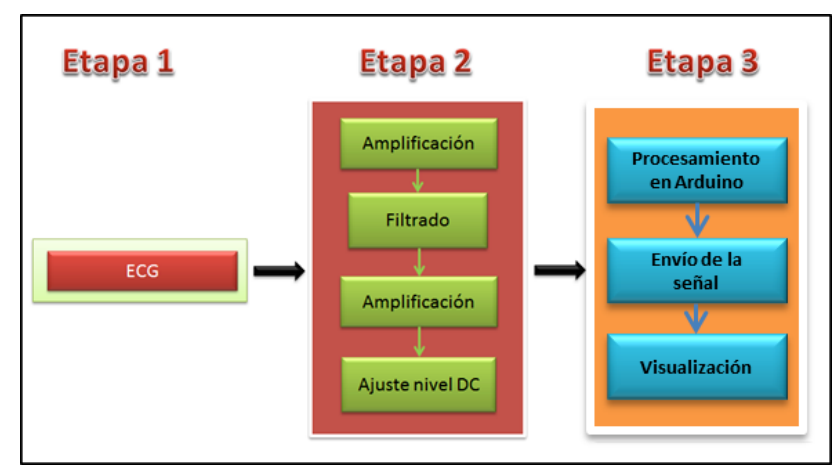

Figura 4. Diagrama de bloques de etapas de diseño.

Fuente: elaboración de los autores.

De forma detallada se explican a continuación las tres etapas usadas en el diseño del presente prototipo de electrocardiógrafo:

\section{Etapa 1: Análisis fisiológico}

Se inicia con un análisis fisiológico de la señal a cuantificar, procesar y visualizar. Dentro de este análisis se determina el tipo de registro a realizar, el cual depende del método para obtener la señal de ECG. Existen diversas formas o métodos para obtener un registro ECG, dentro de los de mayor uso encontramos dos: los métodos invasivos, los cuales se caracterizan por realizar las medidas sobre el tejido del miocardio, exponiendo el corazón a través de la apertura del tórax. El segundo método es no invasivo, en el cual se emplea electrodos superficiales para tomar medidas de impedancia eléctrica, sin producir algún tipo de lesión en el paciente (Salazar, 2004); este último método fue utilizado en este proyecto.

Para la captura de la señal, fue necesario conocer e identificar las señales eléctricas correspondientes a los movimientos que realiza el corazón, con el fin de desarrollar un adecuado tratamiento de la señal. Esta actividad permitió en la siguiente etapa realizar una adaptación óptima, garantizando una correcta medición de los datos y evitando pérdidas significativas de la señal.

\section{Etapa 2: Adaptación de la señal ECG}

Debido a la baja amplitud en voltaje que presenta este tipo de señales, es necesario adaptarlas de tal manera que puedan ser procesadas por el Arduino ${ }^{\circledR}$. En el proceso de acondicionamiento de la señal es necesario tener en cuenta la manipulación que se le realizo a la señal, el uso de filtros y elevador de nivel, también es- 
pecificar el umbral establecido de la frecuencia cardiaca (Becerra, 2007).

Después de terminado la primera fase de amplificación, la señal es filtrada a la frecuencia que maneja cada señal, con el fin de eliminar ruidos externos que puedan afectar la lectura y procesamiento de la señal. En esta fase, se pueden presentar atenuaciones por lo que es necesario amplificar nuevamente la señal. Finalmente, la señal saliente todavía contiene valores de voltaje negativos que no son procesados en el Arduino ${ }^{\varpi}$, por lo cual es necesario realizar un ajuste del nivel DC, para que la señal solo tenga valores de voltaje positivos y puedan ser procesados en la tarjeta Arduino ${ }^{\circledR}$. El acondicionamiento de la señal electrocardiográfica es de suma importancia para referenciar la amplitud de la señal con la entrada al procesador digital, en la Figura 5 se explica paso a paso los valores de amplitud necesarios para acondicionar la señal cardíaca. Nótese que la entrada puede provenir de un paciente real o de un simulador que entrega $1 \mathrm{mV}$ de salida, se realiza una amplificación de instrumentación de 1:1000 y luego una operacional de 1:5 para cambiar la referencia a 2.5 del valor máximo.

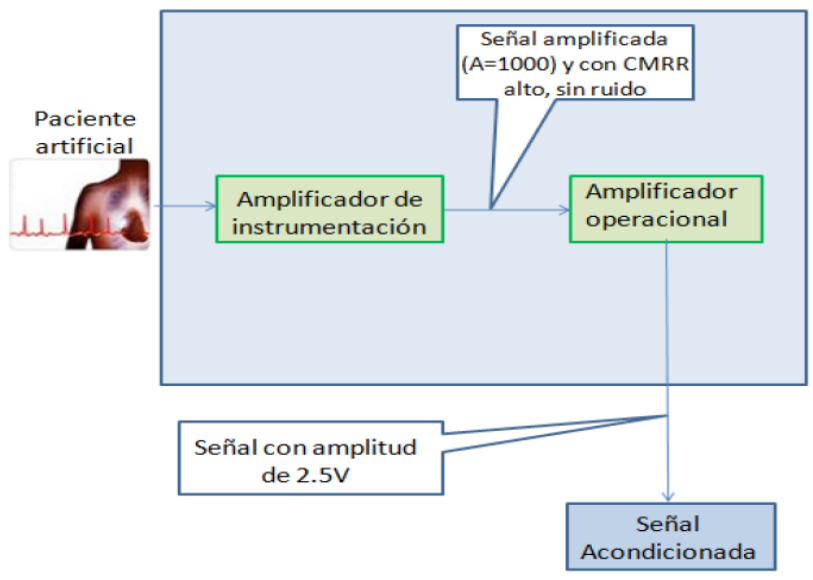

Figura 5. Acondicionamiento de la señal cardíaca.

Fuente: Becerra, J. A. (2007). EGC móvil utilizando tecnología Inalámbrica. Bogotá, Colombia: Universidad Militar Nueva Granada.

\section{Etapa 3: Procesamiento y visualización}

Esta etapa se procesa, envía y visualiza la señal obtenida. En la primera fase del procesamiento de la señal de ECG obtenida se hace en forma digital ya que usualmente es más cómodo de realizar y más barato de implementar que el procesamiento analógico. Además, las señales digitales requieren menos ancho de banda y pueden ser comprimidas. Las señales digitales son manejadas por el microcontrolador de la tarjeta.
El microcontrolador se programó con el Lenguaje de Arduino ${ }^{\oplus}$, para enviar las señales inalámbricamente o por un medio guiado a un dispositivo final. En la fase de visualización se utilizó la interfaz gráfica del dispositivo que recibe las señales, visualizando los valores y las gráficas correspondientes a cada señal.

\section{Resultados}

El ECG académico desarrollado por el grupo TIGUM, fue implementado y validado durante las 3 etapas mencionadas anteriormente. Para la obtención de la señal EGC fue necesario que el circuito de prueba, presentado en la Figura 6, contara con los siguientes elementos un LCD gráfico y con Arduino ${ }^{\circledR}$, un circuito de ajuste de nivel DC para modificar el voltaje y ajustar a partir del potenciómetro implementado en el circuito. En la Figura 6 se muestra el montaje desarrollado a nivel de protoboard, que al ser de uso académico permite realizar diversos cambios en cada una de las etapas y visualizar en tiempo real la señal ECG. Es importante, presentar un ensamble modular que los estudiantes puedan conectar y desconectar y analizar al tratamiento de la señal.

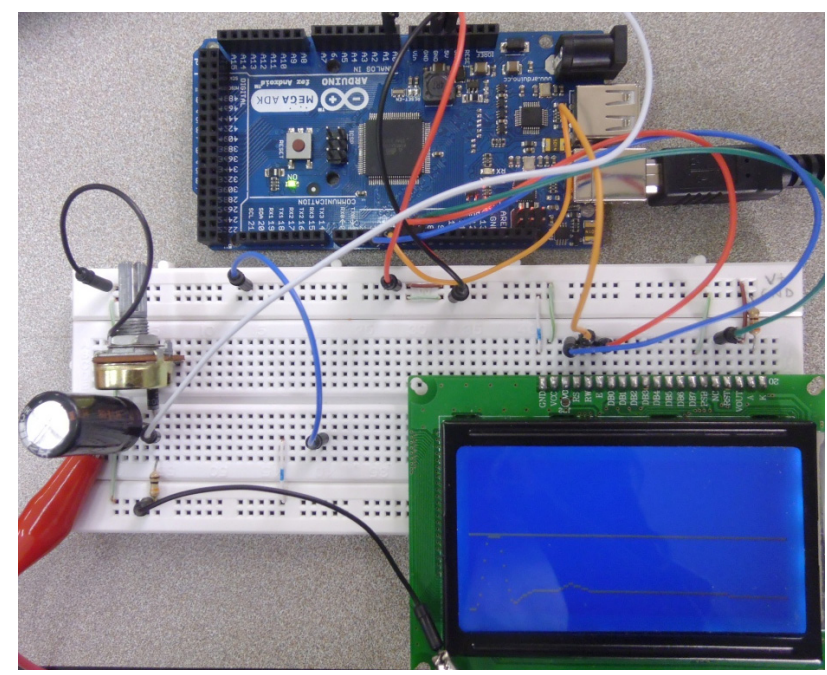

Figura 6. Prueba de visualización señal ECG con LCD Gráfico y Arduino ${ }^{\circledR}$.

Fuente: Los autores.

En la etapa de adaptación de la señal ECG, proveniente del simulador es importante tener en cuenta que existen tres derivaciones de la señal conectadas a la etapa amplificadora y de filtrado mediante tres latiguillos de ECG, que se conectan desde el simulador hasta las entradas de la etapa amplificadora, dichos latiguillos también se conectan desde el paciente real con electro- 
dos, pero en este caso no son necesarios los electrodos ya que este dispositivo envía la señal directamente desde sus salidas a los conectores.

La salida obtenida es una única señal, que debido su naturaleza es necesario conectarla una de las entradas análoga del Arduino ${ }^{\oplus}$, y mediante que el pin análogo convertirla en una señal digital, para luego ser procesada adecuadamente por el Arduino ${ }^{\bullet}$. En la Figura 7 se muestra el módulo de amplificación y filtraje de la señal de ECG ya ensamblada en una tarjeta diseñada por los autores, lo que permite a los estudiantes que realicen su montaje en protoboard, y mediante comparación en cada punto de prueba hacer seguimiento a la amplificación, manejo del ruido y su correspondiente filtraje. Se puede notar que al usar el simulador de paciente, la conexión de los electrodos es directa a su etapa de amplificación, esto difiere al momento de hacerlo sobre un paciente real, cuya piel se suma una resistencia entre los $100 \Omega$ a $500 \Omega$ dependiendo de la cantidad de grasa. Es ese caso, es necesario agregar un gel conductor que evite esta resistencia y elimine la atenuación de la señal de ECG. Para este artículo se presentan resultados del prototipo de ECG diseñado para uso académico, que no revista peligro alguno a los estudiantes y para eso se utiliza la señal desde el paciente simulado por el equipo Fluke PS420.

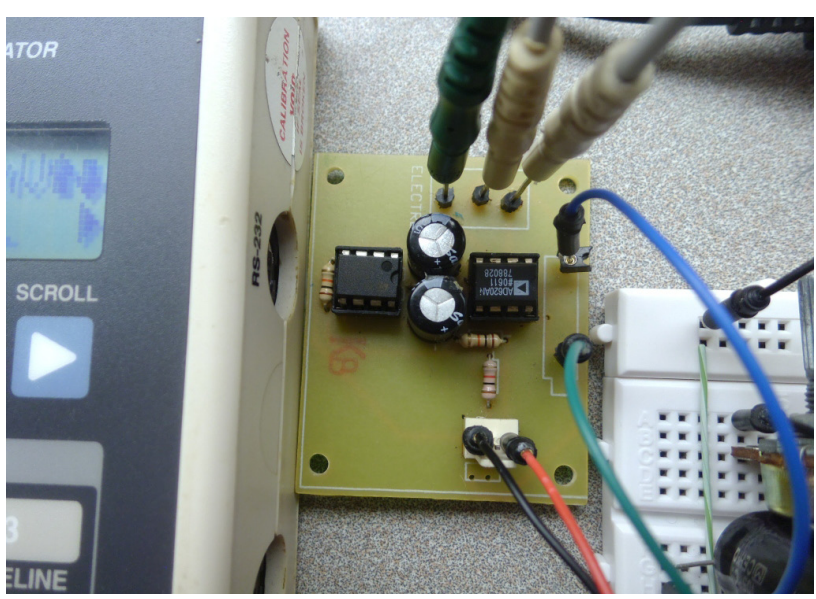

Figura 7. Etapa de amplificación de señal ECG.

Fuente: Los autores

La Figura 8, presenta los resultados de la señal EGC obtenida en una pantalla de cristal líquido. Esta visualización corresponde a la señal del ECG en una baja resolución de la pantalla de cristal líquido. En la parte superior se ha dejado una línea de referencia que permite apreciar la señal, se atenúa, o la etapa de amplificación, la cual está sobreponiendo ruido amplificado que modifique la señal original.

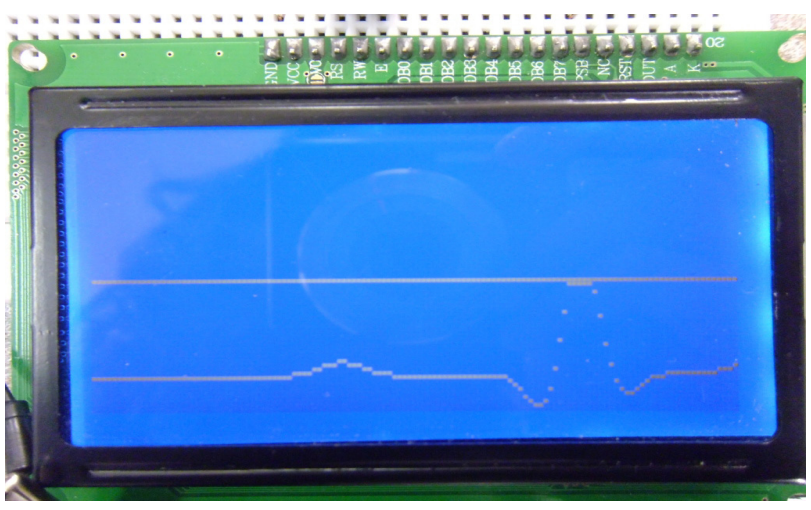

Figura 8. Visualización señal ECG en display gráfico.

Fuente: Los autores.

Por último, la Figura 9, se expone el prototipo completo desarrollado para la adquisición, pre-procesamiento, acondicionamiento, procesamiento y visualización de la señal ECG. El montaje presentado permitió a los estudiantes de ingeniería y de medicina hacer múltiples pruebas en tiempo real a cada etapa del diseño presentado. El prototipo fue probado por 15 estudiantes de ingeniería y 15 estudiantes de medicina, logrando que cada área del conocimiento comprenda desde el análisis fisiológico del corazón el comportamiento, mecánica y la generación de señal eléctrica.

Las evidencias de los estudiantes y sus profesores fue altamente satisfactoria, expresando que:

- Ahora sí puedo entender claramente los valores de amplitud en $\mathrm{mV}$ que nuestro cuerpo genera, desde la actividad cardiaca en la capa superficial de la piel.

- Comprendo el uso de amplificadores, filtros y demás componentes electrónicos en el diseño y puedo cambiar a satisfacción los elementos, logrando visualizar en tiempo real la transformación que sufre la señal de salida.

- Es claro entender el uso de procesadores de señal análogos y digitales que ayuden a cálculos de modelos matemáticos y sistemas de referencia digital (1-0).

- Como médico comprendo el funcionamiento de cada módulo e identifique su operación en el diseño circuital. Antes solo eran diagramas de flujo, ahora es real y podemos ajustar los parámetros de uso.

- Es vital entender el funcionamiento del equipo de electrocardiografía para tener los cuidados y precauciones necesarias al momento de adquirir, registrar y procesar la señal cardiaca. 


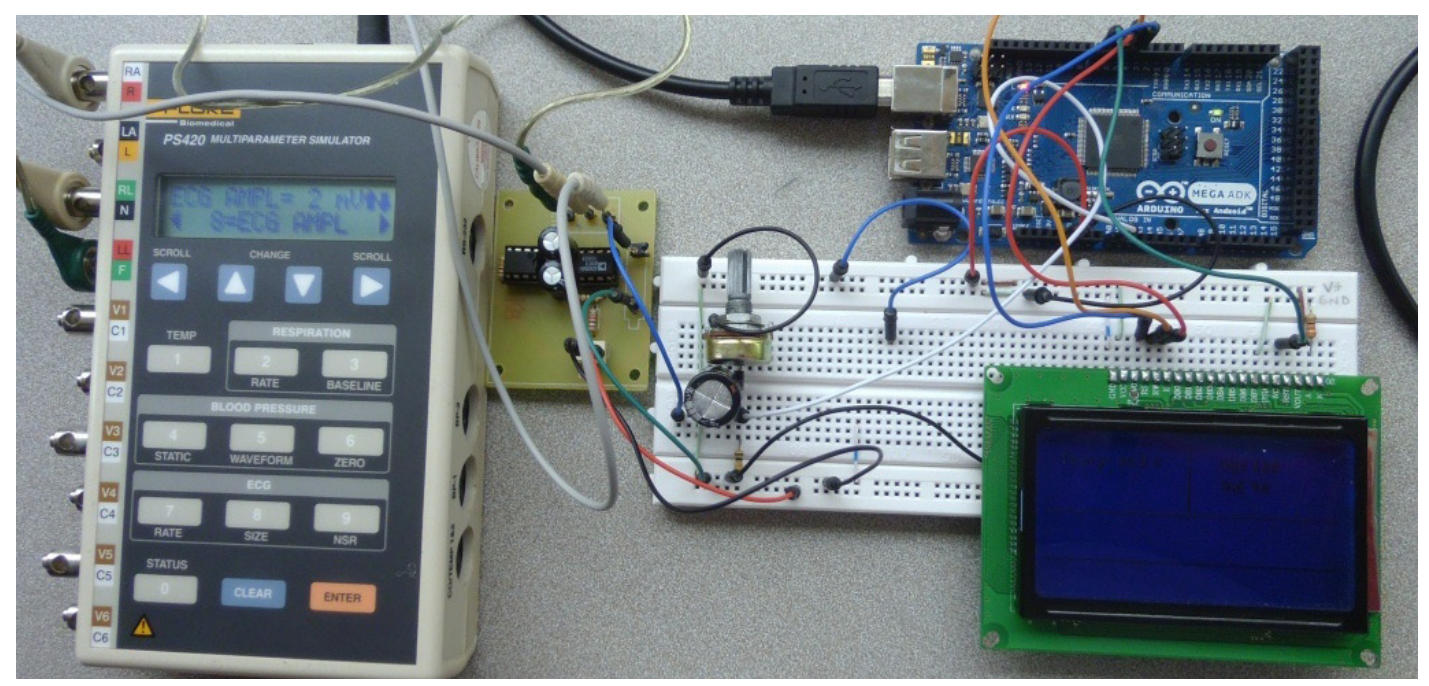

Figura 9. Etapa de adquisición y amplificación de señales ECG.

Fuente: Los autores.

\section{Discusión}

El dispositivo desarrollado con fines académicos es fácil de uso, no invasivo, con respuesta eficiente en el tiempo y cuenta con la capacidad de almacenamiento. Al integrar la plataforma de Arduino ${ }^{\circledast}$ facilita el montaje del sistema y su utilización, puesto que el lenguaje de programación es amigable. También Arduino ${ }^{\circledR}$ es una herramienta libre que permite la creación de múltiples aplicaciones en la electrónica en diferentes campos. Es sencilla, ágil, portable y al no requerir cableado adicional en cuanto a su funcionamiento en general, se puede utilizar por estudiante en la realización de proyectos académicos.

Es vital que el modelo de aprendizaje permita pasar de la teoría a la práctica, especialmente con equipos y dispositivos que midan y registren parámetros de la salud humana, porque su total entendimiento permite al médico, biomédico e ingeniero realizar una operatividad óptima del equipo.

La muestra de treinta estudiantes de diferentes áreas del conocimiento fue importante para valorar el nivel de comprensión de los diferentes módulos del equipo, modo de operación individual y estrategias de integración para adquisición, pre-procesamiento, procesamiento, almacenamiento y visualización.

\section{Conclusiones}

El prototipo de electrocardiógrafo bipolar para uso académico se desarrolló en un montaje práctico, fácil de operar, modular y de bajo costo, que permitió la obten- ción de una señal EGC que comparada con una referencia patrón generada desde un simulador de ECG fluke PS420 muestra una correlación de 0.98 que es significativamente alta.

La etapa de filtrado y acondicionamiento de la señal tiene una gran importancia, debido a la eliminación del ruido producido desde los artefactos y electrodos instalados para la adquisición de la señal. Las etapas posteriores de procesamiento y visualización fueron diseñadas para que los estudiantes hagan diversas prácticas con los valores y tipo de los elementos.

En la etapa de adaptación de la señal es necesario realizar el ajuste de nivel DC para ingresar al procesador sólo valores positivos de amplitud, y generar un programa de procesador en $\mathrm{C}++$ para el Arduino ${ }^{\oplus}$ con referencia único al cero lógico.

Dentro de la investigación realizada se encontró que el equipo de simulación debe ser previamente calibrados y ajustados cada día, esto por el uso prolongado que modifica los parámetros establecidos en su fabricación y esto conlleva a que los datos obtenidos presenten un margen del error del 30\%.

Para concluir, los avances tecnológicos actuales han permitido desarrollar dispositivos electrónicos de prueba a bajo costo, portables y con beneficios para la sociedad en general y principalmente para la academia, facilitando el aprendizaje y la investigación de los estudiantes del funcionamiento del organismo humano. 
El uso académico de este tipo de desarrollos es valorado por los estudiantes y profesores para lograr tener herramientas que lleven de la teoría a la práctica el conocimiento adquirido en las aulas. Hablando de equipo médico, los altos costos impiden tener equipos para prueba de sus componentes. Este tipo de prototipos permite modificar, re-diseñar y operar módulos independientes para otros tipos de señales, ejemplo electromiografías.

\section{Reconocimientos}

Los autores reconocen el apoyo recibido de la Vicerrectoría de Investigaciones de la Universidad Militar Nueva Granada, al proyecto PIC-ING-1668.

\section{Referencias}

Acierno, L. J. (1994). The history of cardiology, pp. 597-697. London; New York: Parthenon Publishing Group.

Arango, J. J., Vélez, H., Rojas, W., Borrero, J., y Restrepo, J. (2003). Fundamentos de Medicina: Manual de Electrocardiografía, 344.Medellín, Colombia: Corporación para Investigaciones Biológicas.

Becerra, J. A. (2007). EGC móvil utilizando tecnología Inalámbrica. Bogotá, Colombia: Universidad Militar Nueva Granada.

Correa, C. A., Bolaños, R. A., y Escobar, A. (2007). Análisis de esquemas de filtrado análogo para Señales ecg. Scientia et Technica, 5 (37).

Edminister, J. A., Nahvi, M., Navarro, R. S., Sánchez, E. L., y De Miguel Rodríguez, P. (1997). Circuitos eléctricos, 3 (2). McGraw-Hill.

Fluke Biomedical PS420. [En línea] Disponible en http:// es.flukebiomedical.com/Biomedical/usen/About/Press/ PS420+press+release.htm. Recuperado el 25 de noviembre de 2014.

L. Finance, C. (1975). El cuerpo humano primera parte: corazón y sistema circulatorio.

Lozano, F. A; Rondón C. R. (2008). Diseño de sistema detector de taquicardia Ventricular y envió de mensaje a celular. Bogotá, Colombia: Universidad Militar Nueva Granada.

Melo, H. y Maya, A. (2009) Dispositivo para telemetría de señales biológicas que permite la utilización de diferentes tecnologías. Bogotá, Colombia: Universidad Militar Nueva Granada.

PROTEUS ISIS52. (2008). Labcenter Electronics Ltd, version 8.1, [CD-ROM]. Programa computacional.

PS420 Simulador de Paciente. [En línea] Disponible en http:// es.flukebiomedical.com/biomedical/usen/Biomedical/FBSIMS/PS420.htm?PID=56628 Recuperado el 25 de noviembre de 2014.

Salazar Muñoz, Y. (2004). Caracterización de tejidos cardíacos mediante métodos mínimamente invasivos y no invasivos basados en espectroscopia de impedancia eléctrica. Barcelona, España: Universidad Politécnica de Cataluña.
Para citar este artículo:

Ramírez, L., Rodríguez, Y., y Cifuentes, Y. (2014). Prototipo de electrocardiógrafo bipolar para uso académico. Ciencia y Poder Aéreo, Vol. 9 (1). 